\title{
Cross-Channel Interference Due to Mode Partition Noise in WDM Optical Systems Using Self-Seeded Gain-Switched Pulse Sources
}

\author{
L. P. Barry, Member, IEEE, and P. Anandarajah, Student Member, IEEE
}

\begin{abstract}
The sidemode suppression ratio of self-seeded, gainswitched optical pulses is shown to be a vital parameter in determining the usefulness of these pulses in wavelength-divisionmultiplexed communications systems. Experiments carried out on a two-channel wavelength multiplexed setup using tunable selfseeded gain-switched pulse sources at $10 \mathrm{GHz}$, have demonstrated the cross-channel interference effects that may be encountered if the sidemode suppression ratio of one of the sources becomes degraded.
\end{abstract}

Index Terms-Gain-switching, optical fiber communications, optical pulse generation, self-seeding, semiconductor laser, wavelength-division-multiplexing.

\section{INTRODUCTION}

$\mathbf{T}$ HE DEVELOPMENT of a wavelength-tunable source of short optical pulses operating at $10 \mathrm{GHz}$ is vital for use in wavelength-division-multiplexed (WDM), optical time-division-multiplexed (OTDM), and hybrid WDM/OTDM optical communication systems [1]. One of the most reliable techniques available to generate wavelength-tunable, picosecond optical pulses involves the self-seeding of a gain-switched Fabry-Perot (FP) laser [2]-[4]. One important characteristic of these sources is the variation in the sidemode suppression ratio (SMSR) as the wavelength is tuned [2]-[4], as this may ultimately affect their usefulness in optical communication systems. In a recent letter [5], we demonstrated how this SMSR variation greatly affected the noise induced on a single $2.5-\mathrm{GHz}$ pulse source as the pulses propagated through optical fiber and an optical filter.

In this letter, we experimentally investigate the effect of the pulse SMSR on the performance of $10-\mathrm{GHz}$ self-seeded gain-switched (SSGS) pulse sources in a two-channel WDMtype system. We examine the noise induced on one of the pulse sources due to a variation in SMSR of the other SSGS source. Our results show that although many of the reported wavelength-tunable pulse sources using the SSGS technique had SMSRs that varied between 10 and $25 \mathrm{~dB}$ as the output pulse wavelength was tuned [2]-[4], in practice, such pulses may be unsuitable for use in high-speed WDM communication systems due to cross-channel interference caused by the mode partition effect [6], [7].

Manuscript received June 28, 2000; revised November 13, 2000.

The authors are with the School of Electronic Engineering, Dublin City University, Dublin 9, Ireland (e-mail: barryl@eeng.dcu.ie).

Publisher Item Identifier S 1041-1135(01)01997-8.

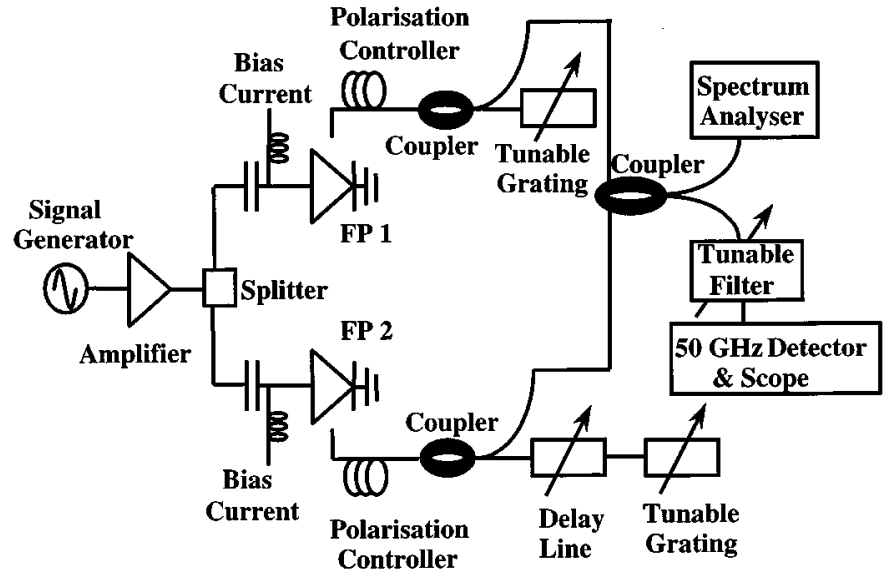

Fig. 1. Experimental setup for examining the effects of SMSR variation in a WDM-type system using two SSGS pulse sources.

\section{EXPERIMENTAL SETUP}

Fig. 1 shows our experimental setup. The FP lasers used were commercial $1.5-\mu \mathrm{m}$ InGaAsP devices, with threshold currents around $25 \mathrm{~mA}$ and longitudinal mode spacings of $1.1 \mathrm{~nm}$. The two lasers used had central frequencies of $1556 \mathrm{~nm}$. Gain switching of the lasers was carried out by applying dc bias currents of around $45 \mathrm{~mA}$, and $10-\mathrm{GHz}$ sinusoidal modulation signals with powers of $24 \mathrm{dBm}$, to each device. Self seeding of the diode FP1 was achieved by using an external cavity containing a polarization controller (PC), a 3-dB coupler, and a tunable Bragg grating with a bandwidth of $0.4 \mathrm{~nm}$. The external cavity for self-seeding FP2 contained an additional tunable optical delay line.

To achieve optimum SSGS pulse generation from FP1, the grating was tuned to reflect one of the laser modes (at $1556 \mathrm{~nm}$ ), and the frequency of the sinusoidal modulation was then varied $(\sim 9.987 \mathrm{GHz})$ to ensure that the signal reinjected into the laser arrives at the correct time. For SSGS operation of FP2, the Bragg grating was tuned to reflect a laser mode at $1546 \mathrm{~nm}$, and the optical delay line was varied to ensure that the signal fed back from the grating arrives at the correct time. It should also be noted that the wavelength of each source may be tuned using the fiber grating, but the tuning range was limited to about $5 \mathrm{~nm}$ by the tunability of the grating. In addition to tuning the grating, and adjusting the sinusoidal frequency, the feedback can be adjusted, (and thus the SMSR on the output pulses varied), by using the polarization controllers (PC). 


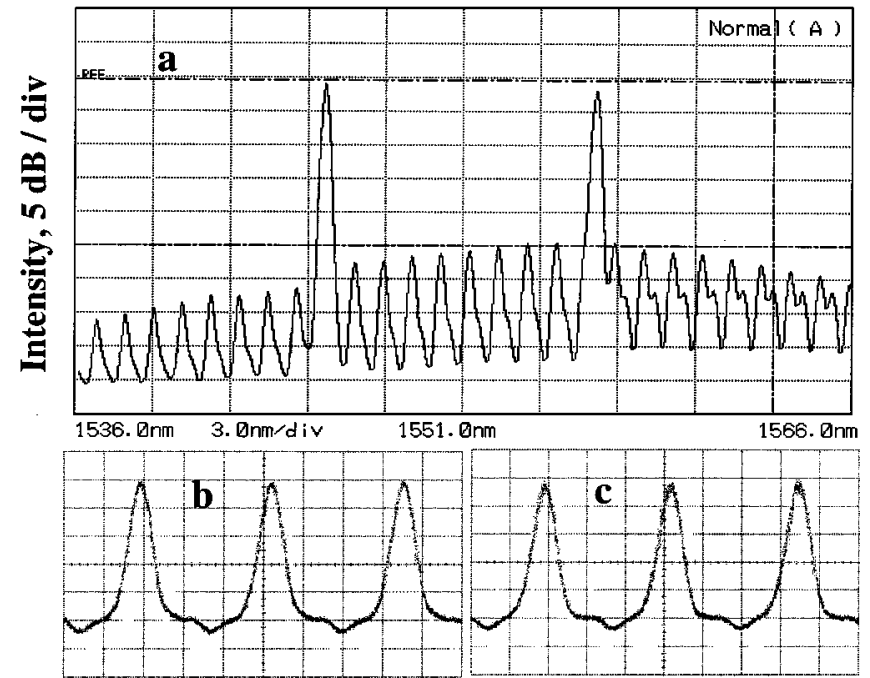

Time, 30 ps / div

Fig. 2. (a) Optical spectrum of the two $10-\mathrm{GHz}$ pulse sources after fiber coupler. (b) and (c) 10-GHz pulse trains of the 1546- and 1556-nm sources (nonaveraged).

\section{RESULTS}

Fig. 2(a) displays the optical spectrum of the two pulse sources after being combined together using a fiber coupler, (with the feedback from the gratings optimized using the polarization controllers). The $3-\mathrm{dB}$ bandwidths of the $10-\mathrm{GHz}$ pulse sources were both around $0.25 \mathrm{~nm}$, and the SMSR of the sources at $1546 \mathrm{~nm}$ and $1556 \mathrm{~nm}$ were $27 \mathrm{~dB}$ and $25 \mathrm{~dB}$, respectively [these SMSR values have been obtained by examining the spectral output from each source independently, and not from the composite signal shown in Fig. 2(a)]. Fig. 2(b) and (c) displays the two pulse waveforms from the sources when they were subsequently filtered out from the composite signal using a tunable FP filter with a bandwidth of $0.7 \mathrm{~nm}$. This particular filter bandwidth is chosen as it is narrow enough to select only a single mode from the optical signal, but large enough such that it does not affect the shape of the optical pulses passing through it. The pulses were detected and measured using a $50-\mathrm{GHz}$ pin photodiode in conjunction with a $50-\mathrm{GHz}$ digitizing oscilloscope. The output pulse duration was $18 \mathrm{ps}$ for the 1546-nm source, and $19 \mathrm{ps}$ for the 1556-nm source.

To determine the effect of SMSR on the filtered signals, we initially varied the SMSR of the 1556-nm pulses using the PC, and examined the noise added to the filtered signal at $1546 \mathrm{~nm}$ (which had its SMSR maintained at $27 \mathrm{~dB}$ ). We should point out that the pulses from the two sources are temporally overlapped, thus the interference from one source is directly on top of the adjacent source in our results. Fig. 3(a), (b), and (c) displays the nonaveraged waveform of the filtered 1546-nm signal with the SMSR of the $1556-\mathrm{nm}$ pulse train set to 20,15 , and $10 \mathrm{~dB}$. We can clearly see that when the SMSR was reduced to $15 \mathrm{~dB}$, the noise on the pulse train after the optical filter became noticeable, and as the SMSR was reduced further, the noise on the signal greatly increased. We then examined the effect of varying the SMSR of the 1546-nm source using the PC, when the FP optical filter was tuned to select out the 1556-nm pulse train (which had its SMSR maintained at $25 \mathrm{~dB}$ ). Fig. 4(a) and (b) displays the

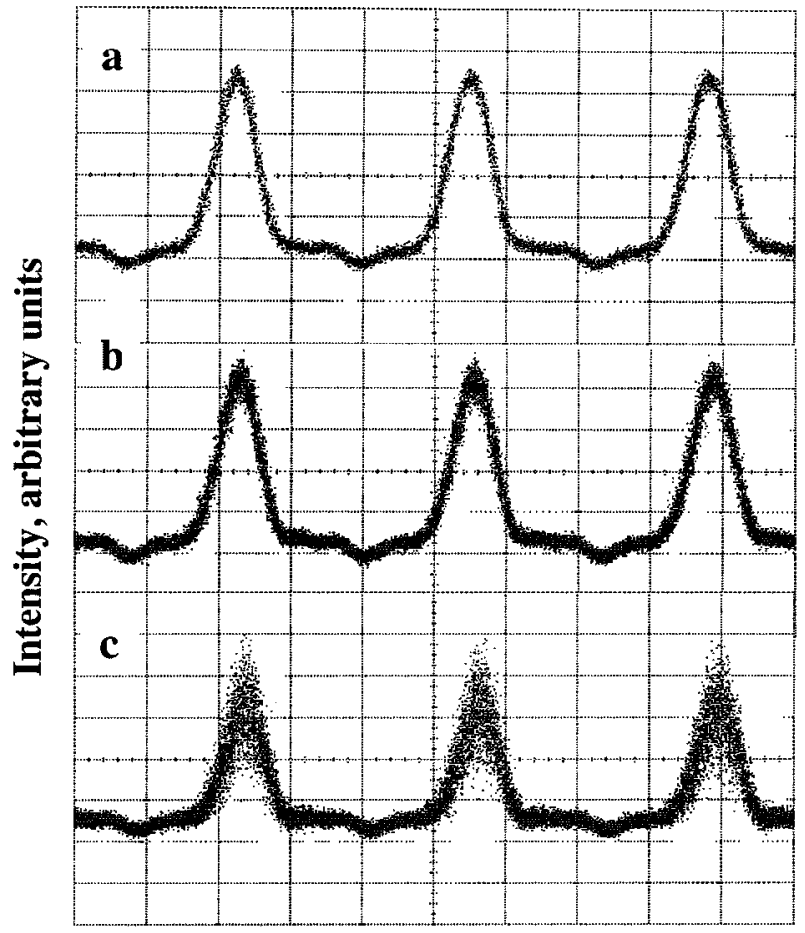

Time, 30 ps / div

Fig. 3. (a), (b) and (c) 1546-nm pulses after FP filter with the SMSR of the 1556-nm pulse source set to (a) 20 , (b) 15 , and (c) $10 \mathrm{~dB}$.

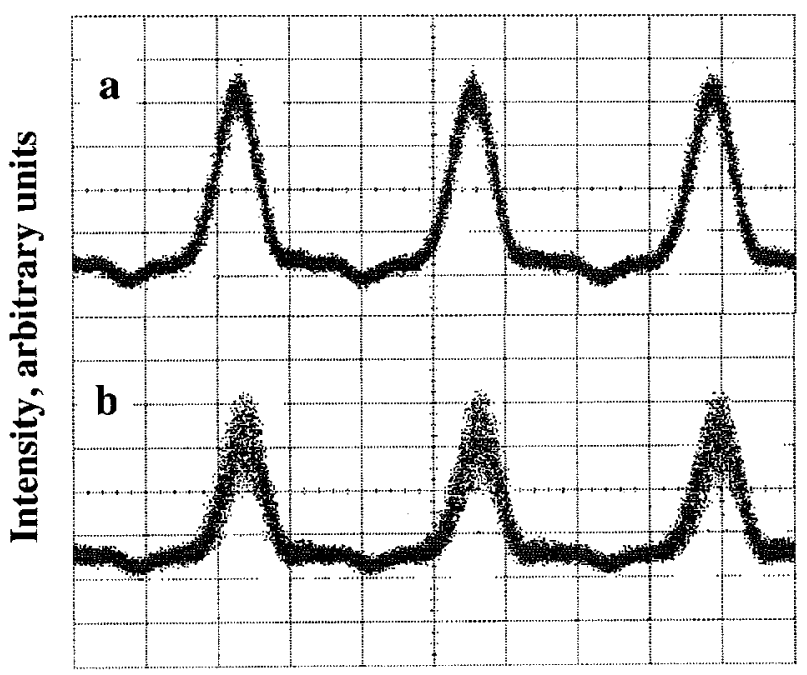

Time, 30 ps / div

Fig. 4. (a) and (b) 1556-nm pulses after FP filter with the SMSR of the 1546-nm source set to (a) $20 \mathrm{~dB}$, and (b) $15 \mathrm{~dB}$.

filtered 1556-nm pulse train when the SMSR of the 1546-nm signal was set to 20 and $15 \mathrm{~dB}$. We can clearly see that the noise level on the signal increases as the SMSR of the 1546-nm source was reduced.

We subsequently investigated how the mode-partition-noise was affected by varying the spectral spacing between the two sources. In this case, the amplitude noise on the detected pulse was characterized by measuring its rms noise voltage using the digitizing oscilloscope. Fig. 5 displays the results when the SSGS source using FP2 was tuned from 1543.8 to $1548.2 \mathrm{~nm}$ 


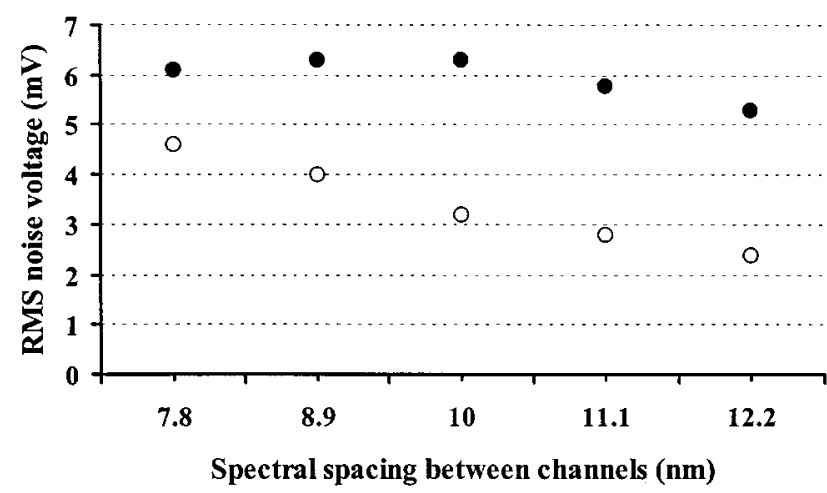

Fig. 5. Rms noise voltage (due to cross-channel interference) on detected pulses from FP1 $(\bullet)$ and FP2 (o), as their wavelengths are tuned over 5 FP modes, with the SMSR of the adjacent pulse source (fixed wavelength) set to $15 \mathrm{~dB}$.

(with its SMSR kept constant around $27 \mathrm{~dB}$ ), and the SMSR of the SSGS at 1556 was set to $15 \mathrm{~dB}$. We can see that as the spectral spacing decreases, the noise level on the filtered out signal from FP2 increases. We then tuned the pulse source from FP1 between 1553.8 and $1558.2 \mathrm{~nm}$ (with its SMSR kept constant around $25 \mathrm{~dB}$ ), and examined the interference due to the 1546-nm SSGS source from FP2 (with its SMSR set to $15 \mathrm{~dB}$ ). These results are also shown in Fig. 5, and demonstrate that in this case the noise level on the filtered out pulse is actually maximum when it is at $1556 \mathrm{~nm}$ (approximately 10-nm spacing between the SSGS sources), and decreases slightly as the source is tuned to higher or lower wavelengths.

\section{DISCUSSION AND CONCLUSION}

The noise acquired on a filtered pulse source as the SMSR of the other SSGS pulse source is reduced, is due to the mode partition effect [6], [7]. This effect is basically a fluctuation of the energy in each laser mode with time, due to a constant transfer of energy between the modes. For a single-mode laser with a large SMSR, the power in the side modes is negligible, thus the power fluctuation of the main mode is negligible. However, as the SMSR decreases, the power fluctuation of the main mode, and the side modes, may become nonnegligible. When the optical filter is tuned to select out either the 1546- or 1556-nm signal, the noise on the pulse train will be negligible provided the SMSRs of both sources is large [as shown in Fig. 2(b) and (c)]. If the SMSR of the filtered signal is reduced, then the noise level on that pulse source would clearly increase due to the mode partition effect, as already demonstrated and explained in [5]. However, in this experiment we have demonstrated the increased noise induced on one filtered pulse source due to a reduction in SMSR of a second SSGS source. This noise is also due to the mode partition effect, because as the SMSR of one
SSGS pulse source is reduced, the power (and the power fluctuation) in its side mode, which is at the same wavelength as the second pulse source selected by the FP filter, increases. The temporal fluctuation in power of this side mode can thus manifest itself as noise on the filtered source.

In order to explain the variation in amplitude noise as the spectral spacing between the sources is varied, it is necessary to understand that the cross-channel interference (caused by mode-partition-noise) on channel 1 due to channel 2 is determined by the power in the side mode of channel 2, which is at the same wavelength as channel 1 . The power in the side mode of any SSGS pulse source (using an FP laser) is determined by the spacing between the side mode and the peak of the FP gain curve. Thus, as the wavelength of channel 1 is varied, the cross-channel interference due to channel 2 is determined by the position (wavelength) of channel 1 relative to the gain curve of the FP laser used to generate the SSGS pulses for channel 2.

In conclusion, we have shown that the SMSR of wavelengthtunable SSGS pulse sources at $10 \mathrm{GHz}$ is extremely important for determining their usefulness in WDM communication systems. If the SMSR of one source in a WDM system becomes degraded, then the interaction of the mode partition effect with spectral filtering can result in a large amount of noise on all the wavelength channels in the communications system. This noise could clearly lead to an unacceptable error rate in the communication system. It is thus vital that any WDM transmission system based on tunable SSGS pulse sources maintains a large SMSR (preferably greater than $30 \mathrm{~dB}$ ) at all wavelengths.

\section{REFERENCES}

[1] T. Morioka, H. Takara, S. Kawanishi, O. Kamatani, K. Takiguchi, K. Uchiyama, M. Saruwatari, H. Takahashi, M. Yamada, T. Kanamori, and H. Ono, "1Tbit/s (100Gbit/s times 10 channel) OTDM/WDM transmission using a single supercontinuum WDM source," Electron. Lett., vol. 32, pp. 906-907, May 1996.

[2] L. P. Barry, R. F. O' Dowd, J. Debeau, and R. Boittin, “Tunable transform limited pulse generation using self-injection locking of a FP laser," IEEE Photon. Technol. Lett., vol. 5, pp. 1132-1134, Oct. 1993.

[3] M. Schell, W. Utz, D. Huhse, J. Kassner, and D. Bimberg, "Low jitter single mode pulse generation by a self-seeded, gain-switched Fabry-Perot semiconductor laser," Appl. Phys. Lett., vol. 65, pp. 3045-3047, 1994.

[4] C. Shu and S. P. Yam, "Effective generation of tunable single- and multi-wavelength optical pulses from a Fabry-Perot laser diode," IEEE Photon. Technol. Lett., vol. 9, pp. 1214-1216, Sept. 1997.

[5] L. P. Barry and P. Anandarajah, "Effect of side mode suppression ratio on the performance of self-seeded, gain-switched optical pulses in lightwave communications systems," IEEE Photon. Technol. Lett., vol. 11, pp. 1360-1363, Nov. 1999.

[6] N. H. Jensen, H. Olesen, and K. E. Stubkjaer, "Partition noise in semiconductor lasers under CW and pulsed operation," IEEE J. Quantum Electron., vol. QE-23, pp. 71-79, 1987.

[7] D. Curter, P. Pepeljugoski, and K. Y. Lau, "Noise properties of electrically gain-switched $1.5 \mu \mathrm{m}$ DFB lasers after spectral filtering," Electron. Lett., vol. 30, pp. 1418-1419, 1994. 